\title{
EVALUATION OF PARTIALLY OVERLAPPING 3D POINT CLOUD'S REGISTRATION BY USING ICP VARIANT AND CLOUDCOMPARE
}

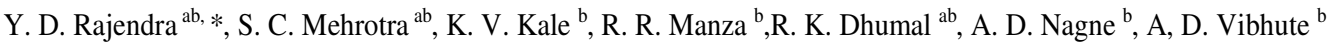 \\ ${ }^{a}$ Srinivasa Ramanujan Geospatial Chair, \\ *yogesh.rajendra@gmail.com,mehrotra.suresh15j@gmail.com,dhumal19@gmail.com \\ ${ }^{\mathrm{b}}$ Dept. of CS \& IT, Dr. B. A. M. University, Aurangabad, MS, India \\ kvkale91@gmail.com, manzaramesh@gmail.com, ajay.nagne@gmail.com, amolvibhute2011@gmail.com
}

\section{Commission VIII, WG VIII/8}

KEY WORDS: TLS, Laser Scanning, Point Cloud, Matching, ICP, Error, Visualization.

\begin{abstract}
:
Terrestrial Laser Scanners (TLS) are used to get dense point samples of large object's surface. TLS is new and efficient method to digitize large object or scene. The collected point samples come into different formats and coordinates. Different scans are required to scan large object such as heritage site. Point cloud registration is considered as important task to bring different scans into whole 3D model in one coordinate system. Point clouds can be registered by using one of the three ways or combination of them, Target based, feature extraction, point cloud based. For the present study we have gone through Point Cloud Based registration approach. We have collected partially overlapped 3D Point Cloud data of Department of Computer Science \& IT (DCSIT) building located in Dr. Babasaheb Ambedkar Marathwada University, Aurangabad. To get the complete point cloud information of the building we have taken 12 scans, 4 scans for exterior and 8 scans for interior façade data collection. There are various algorithms available in literature, but Iterative Closest Point (ICP) is most dominant algorithms. The various researchers have developed variants of ICP for better registration process. The ICP point cloud registration algorithm is based on the search of pairs of nearest points in a two adjacent scans and calculates the transformation parameters between them, it provides advantage that no artificial target is required for registration process. We studied and implemented three variants Brute Force, KDTree, Partial Matching of ICP algorithm in MATLAB. The result shows that the implemented version of ICP algorithm with its variants gives better result with speed and accuracy of registration as compared with CloudCompare Open Source software.
\end{abstract}

\section{INTRODUCTION}

\subsection{Overview}

For several years now, Terrestrial Laser Scanning (TLS) has become an additional surveying technique in geodesy. Recent developments have improved several aspects of terrestrial laser scanners, e.g. the data acquisition rate, accuracy and range.

There is no doubt that remote sensing technology has created a dramatic shift in the past few years concerning how scientists and researchers gathered to analyze information about the Earth. Remote sensing, the use of satellites or aircraft to gather data about objects from a distance, has an almost infinite number of applications. This kind of technology has been used to monitor the environment, map the ocean, and explores the Polar Regions and much more. Now, a form of remote sensing technology called Light Detection and Ranging (LiDAR) is being used to lead a revolution to understand human activity of the past.

The digital preservation of Architectural information, historical monuments using advanced 3D measurement technologies is becoming an efficient tool for study and research purpose in recent decade. In addition to traditional methods such as simple hand measurement and tachometry, 3 Dimensional Terrestrial Laser Scanning (3D TLS) is rapidly becoming one of the most commonly used techniques due to its completeness, accuracy and fastness characteristics for data acquisition (Ong Chee Wei, 2010).
Acquiring surface data with Terrestrial Laser Scanners (TLS) have been improved over recent years. The TLS is becoming advanced and cost-effective and getting ready to enter many engineering geodesy applications. It allows for fast and reliable generation of millions of 3D points for very complex structural environments. There is no universal Terrestrial Laser scanner for all applications (Ismail Abd El Hamid Mohamed, 2008).

The need of digital earth for representing information of architecture in three dimensional forms can play very important role in preservation and renovation. Recent development in Terrestrial Laser Scanning (TLS) proves an efficient way to model and analyze this information. This research evaluates the capability of TLS technology for documenting an urban building in 3D environment. The building of Department of Computer Science \& IT (DCSIT) located at the university campus of Dr. Babasaheb Ambedkar Marathwada University, Aurangabad, (MS), India, has been taken as a case study for the purpose. A 3D point cloud data with colorized intensity has been captured using FARO focus 3D laser scanner. The registration of 3D point clouds data by the Iterative Closest Point (ICP) algorithm implemented in MATLAB Further processing done by using the FARO Scene software and CloudCompare Open Source Software. The developed visualization model may be used to plan interior renovation and interior decoration purpose.

\footnotetext{
* Corresponding author. Y. D. Rajendra, JRF, Srinivasa Ramanujan Geospatial Chair, (yogesh.rajendra@gmail.com).
} 


\subsection{Terrestrial Laser Scanning}

Terrestrial Laser Scanning is an active remote sensing technology that can be used for acquisition of a dense set of three-dimensional (3D) points on a large object or surface (Lichti, D. D., 2000). The TLS is a surveying instrument that massively captures coordinates of ground points in $3 \mathrm{D}$ with high velocity and accuracy. Figure 1.1 shows working principle of phase based and time of flight laser scanner. Laser scanning technology and digital close-range Photogrammetry are two types of technology that can produce 3D model. The main difference between these two technologies is that the equipment that is used and the work procedure. Close range Photogrammetry is a visual method where the camera orientations need to be solved first before capturing an image (Ordóñez, C., Riveiro, 2010). Meanwhile, like most of surveying devices, TLS need to be calibrated periodically (Santala, J., 2010). TLS has become popular in the 1990s for mobile robot navigation (Singh, S., 1991), in the construction of metric scale 3D models, such as sculptures (Beraldin, J.A., 2000) and industrial applications (Sequeira, V., 2003).

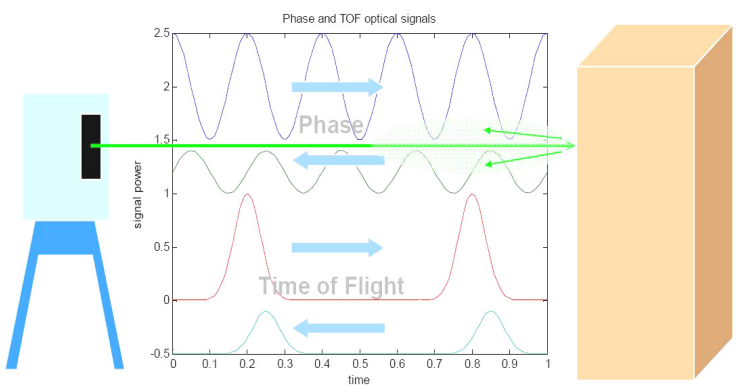

Figure 1.1: Image courtesy of the UC Davis AHMCT Research Center: http://www.ahmct.ucdavis.edu

There are three types of laser scanner systems based on principles of (i) Time of Flight (TOF) (ii) Triangulation (iii) and Phase Shift measurement system (Anne Chung Wei Lin., 2009). TOF measurement system has a laser diode that sends a pulsed laser beam to the scanned object. The pulse is reflected by the surfaces and part of the light will return to the receiver. The time that light travel from laser diode to the object surface and back is calculated. Therefore, the distance to object using assumed speed of light can be calculated from the time that the beam travels (W. Boehler, 2003).

The triangulation system is an active system that uses laser light to probe the environment. Phase shift measurement is a technique for measuring distances that uses a laser beam with sinusoidal modulated optical power being sent to a target. A reflected beam is monitored, and the phase of the power modulation is compared with the emitted beam (Nur Adlina Bt Ramli., 2010). Digital Photogrammetry techniques for object capturing are already well established (Remondino, F., 2006). Image-based technique has a simple data acquisition procedure but it has the limitation on capturing complex surface.

In contrast, laser scanning technique does not bother with the surface shape (Boehler, W., 2004). It also accomplishes the needs of high density of data, speed of capturing and accuracy in different field (Cabrelles, M., 2009). Unfortunately, the laser ray cannot identify the colour of the measured surface. As the result, the obtained 3D point clouds from the laser scanner are colourless. Thus, in most cases, a proper combination of both laser scanning and Photogrammetry technique able to produce better 3D textured model when the characteristics of the study area are complex and with large dimensions (Guarnier, A., 2006).

According to (El-Hakim S., 2002), the generation of detailed 3D models of buildings and artefacts has to accomplish some specifications and requirements in term of geometric accuracy and level of details. In order to get an accurate 3D point cloud data of the whole object surface there are two factors that need to be concerned such as distance accuracy and space resolution of the laser scanner (Pfeifer, N., 2007). Another important issue is about the quality of the intensity value and a feasible influence on distance measurement using laser scanner (Yastikli, N., 2007). The latest developments in sensor technology, laser scanning, offers a new efficient data collection method for measuring an objects. The use of TLS for architectural recording and documentation is becoming increasingly popular (Library of Congress, 2014).

\section{METHODOLOGY}

\subsection{Study Area}

This research was done for the purpose to build a three dimensional modeling of building from 3D point clouds data. The building involved in this research was a Department of Computer Science and Information Technology, Dr. Babasaheb Ambedkar Marathwada University, Aurangabad, (MS), India, as shown in Figure 3.1. At the initial stage of the present study, a collection of research work, related to Terrestrial Laser Scanning, Point Cloud Processing, Iterative Closest Point Algorithm was examined extensively, so that to prepare proper methodologies to assist in the various phases of study.

Figure 2.1: The Site located in Aurangabad, (MS), India.

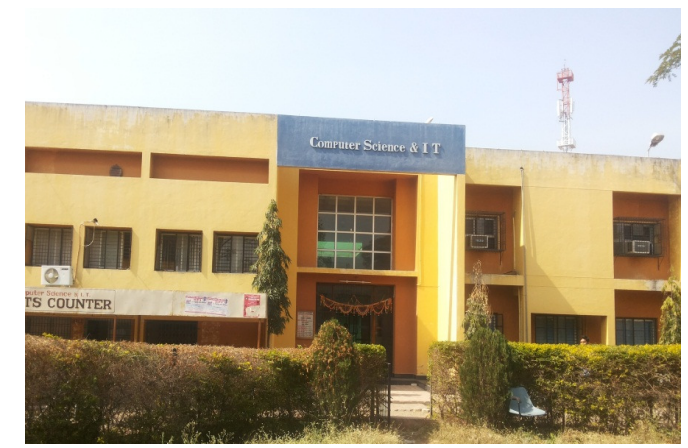

Using a laser scanner to record a building is not just pressing a button and waiting for the deliverables to come out. It requires a profound knowledge of the equipment and the various processes. Some of the steps of the scanning process are quite automated while others are still labour intensive (Sajid Hussain, 2007). This paper will discuss the methodology and steps required to survey a site, scan building using terrestrial laser, collecting point cloud, registration of point cloud using algorithm.

\subsection{Applied Methodology}

The need for 3D digital models is increasing day after day. They are becoming economically convenient to some extent in diverse fields and applications such as visualization, animation, navigation and virtual city models. In particular, 3D photo-realistic modeling is desired for the 3D recording and preservation of sites. These models play an important role in case of loss or damage, tourism, education and museum purposes (P. Dorninger, 2013). The requirements specified for several applications, mainly 3D recording, involve generating high quality 3D models in terms of completeness, geometric accuracy and photo-realistic appearance (D. D. Lichti, 2000). Under that, the processing chain of generating these models comprises five well-known steps: Survey Planning, Field Operation, Data Preparation, Data Registration and Data 
Processing. Figure 2.2 shows block diagram of basic steps required to achieve objectives.

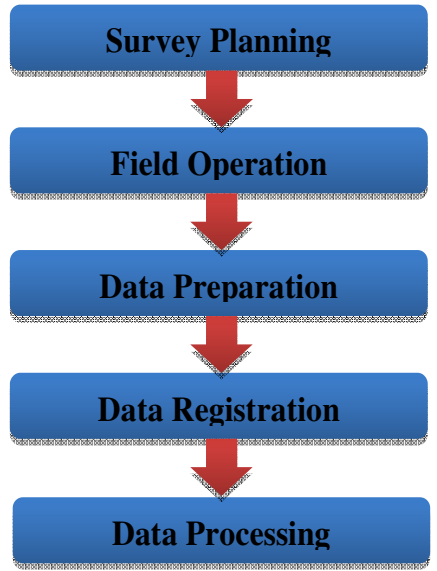

Figure 2.2: Block Diagram of basic steps.

\subsubsection{Survey Planning:}

At present there is no standard procedure for survey planning for Façade Data (Interior + Exterior) using terrestrial laser scanning. However, according to the laser scanning user's community, the survey planning should at least contain the following topics

- Determining the goals and objectives.

- $\quad$ Analyzing the area to be surveyed.

- Determining the measuring techniques and equipment.

- Data management.

Before the laser scanning was done, a reconnaissance work must be carried out first at the field. This was done by moving around the building to make sure there are no obstacles that would block laser scanning process later on and also to determine the best laser scan station's position. Furthermore, the placement of the laser scan station was very crucial to ensure full coverage of the building with a minimum number of stations can be done. If the planning not done correctly, the data

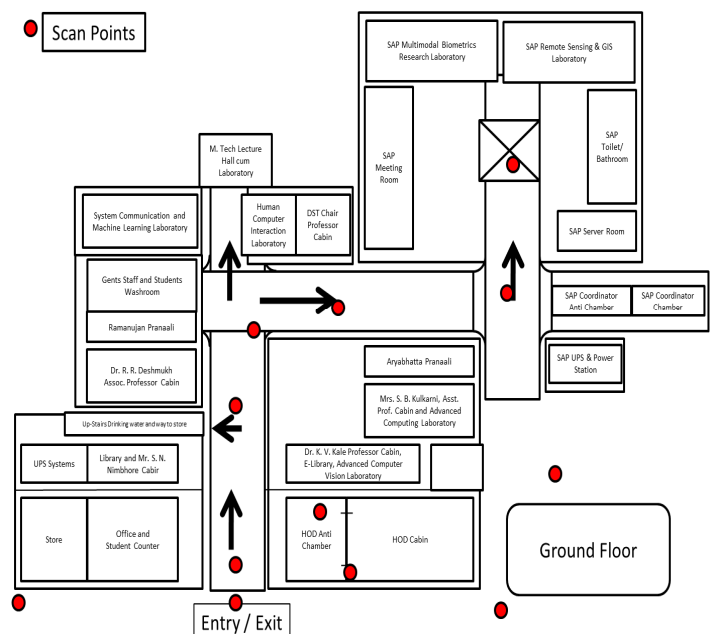

Figure 2.3: Scan positions established to collect interior and exterior information of building.

-obtained later maybe incomplete and rendered useless (G. Vacca, 2012). For the complete site survey we decided a total of 12 laser scan stations See Figure 2.3 were established around and inside the building. This scan station must be reciprocally visible to each other. These stations are considered enough to get full coverage and details of the building based on its size and complexity. The artificial spheres can be used as a target in the scanning process (Nik 'Azim Hazman Bin Nik Ab Hadi, 2011). It works as the control point for the laser scan station. It is very crucial to plan the location of the scan stations in order to ensure that the object and also the spherical target are visible and clearly seen from the various stations.

2.2.2 Field Operation:

The field operation at the DCSIT was carried out with the phase-shift based terrestrial laser scanner FARO Focus 3D. The data capturing procedure was executed in two steps; laser scanning and photographing see Figure 2.4. The geometry and the intensity of façade interior and exterior data were captured by the terrestrial laser scanner while the RGB values of the geometric object were captured by a high resolution digital camera included in FARO Laser Scanner. The DCSIT had very complex features for its inner and outer shape. Hence, an appropriate scanning positions need to be established to capture the full coverage of the façade data (Y. D. Rajendra, 2013).

Laser Scanner FARO Focus 3D system, which uses laser, capable of capturing up to 976,000 points per second. The maximum captured range for this scanner is 153 meters with low ambient light on 90 percent reflective surface. Provides a larger field of view of 360 degrees in horizontal direction and 300 degrees in vertical direction with 0.009 degrees accuracy, and allows the collection of full panoramic views, including color camera of 70 megapixels.

The scanning process is almost fully automated. After pressing the scan button in the scan control software or directly on the scanner control, the scanner moves to the starting point and starts collecting points. These points are stored by the laptop or in the internal memory of the scanner itself. When a laptop is connected to the scanner, the points scanned are directly visualized in three dimensions on the screen and provide an overview of the area that has already been scanned. After scanning, it is a good practice to check the scan for unforeseen obstructions that cause occluded areas in the scan data (Chee Wei, 2010).

Figure 2.4: Faro Focus 3D Terrestrial

$$
\text { Laser Scanner. }
$$

\subsubsection{Data Preparation:}

After the scanning process, data collected by laser scanner needs to take backup. It is advisable to start working on a copy of the original scans and keep the originals as backup. Different types of scanners store the point cloud information in different formats. More information about point cloud file types and their advantages or disadvantages can be found in various literatures (F. Remondino, 2011). For archiving purposes, it is important that the file format is easily accessible and recognizable. If it can be accessed directly, without any decoding, it can later be easily converted to any other format readable by an appropriate piece of software. The file format should also contain the data in its most basic format, instead of using the most preferred format for reprocessing. The most common format at this time (circa 2008) is the *.xyzrgb format. Faro collects data in .fls file format. Before proceeding to process the point clouds, scans affected by extreme environmental conditions or erroneous scans due to human mistakes needs to be removed from the data set. In some cases, it is also required to clean some scans before the registration phase (Renyi Lium Guigang Shi, 2010).

\subsubsection{Data Registration:}

A Registration is only related to rotation and translation of the rigid transformation. A set of point cloud data 
rigid transformation can be expressed as rotation matrix $\mathrm{R}$ and translation matrix $\mathrm{T}$, which makes the point cloud only transform the location and distance. In the three-dimensional space, the rotation matrix $\mathrm{R}$ and translation matrix $\mathrm{T}$ can be expressed as:

$$
\begin{gathered}
R=\left[\begin{array}{ccc}
\cos \alpha & -\sin \alpha & 0 \\
\sin \alpha & \cos \alpha & 0 \\
0 & 0 & 1
\end{array}\right]\left[\begin{array}{ccc}
\cos \beta & 0 & \sin \beta \\
0 & 1 & 0 \\
-\sin \beta & 0 & \cos \beta
\end{array}\right]\left[\begin{array}{ccc}
1 & 0 & 0 \\
0 & \cos \gamma & -\sin \gamma \\
0 & \sin \gamma & \cos \gamma
\end{array}\right] \\
T=\left[\begin{array}{lll}
t_{x} & t_{y} & t_{z}
\end{array}\right] I
\end{gathered}
$$

Where $\alpha, \beta, \gamma$ express rotation angle along $\mathrm{X}, \mathrm{Y}, \mathrm{Z}$ axis, tx, ty, tz express the amount of displacement.

Data registration is considered an important step to get the whole 3D model in one scan world coordinate system for the scanned object while one scan is not enough to describe an object geometrically. Data registration can be described mathematically by a rigid body transformation (M. Hebel, 2007). Data could be registered based on one of three concepts or a combination of them as shown in Figure 2.5.

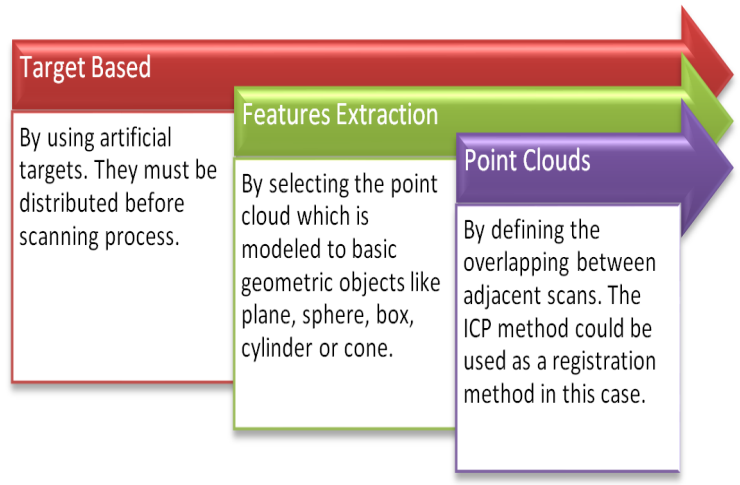

Figure 2.5: Three ways of data registration.

In this work we used point cloud registration approach using basic Iterative Closest Point algorithm. The point cloud processing stage involves the basic checking of data, removal of bad points from point cloud scan caused by blocking object or false returns. For the processing of 12 scan point cloud data we have gone through various phases. The registration involved point clouds from a total of 12 scans location covered the whole DCSIT structure with planned scan direction. There were 4 scans positions at the exterior part and 8 scans in the interior part of the DCSIT. These point clouds have to be registered into one coordinate system in order to achieve a complete visualization model of the Department. Millions of points have been merged to get an integrated view. For the registration and merging purpose we have implemented Iterative Closest Point algorithm in MATLAB for this we required .xyz file format. The .xyz file format is standard format for storing 3D point information by using ASCII values. All the point cloud files having .fls file format are exported to .xyz format so that the processing to be done by using MATLAB and CloudCompare. For the exporting operation we had gone through sub sampling method so to decrease the processing load. Following Figure 2.6 shows point cloud data of building. Data registration based on point clouds without using artificial or natural targets is possible to achieve (Yonghuai Liu, 2006). With this data registration technique all the adjacent scans must be overlapped during the scanning step.

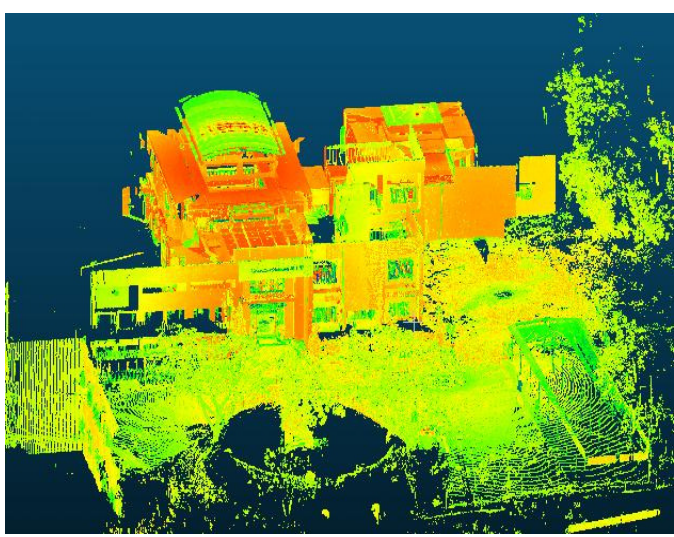

Figure 2.6: Represents all scanned point clouds combined in CloudCompare.

Many techniques like Normal Distributions Transform (NDT), Helmert Transformation, Rigid Body Transformation, etc. could be used to calculate transformation parameters between adjacent scans. These techniques depend on establishing a set of corresponding points from two data sets, after that calculate the transformation parameters based on the corresponding points (Zhang,1994), (Chen, 1991), (Besl, P. 1992). The ICP data registration technique is based on the search of pairs of nearest points in the two adjacent scans and calculates the transformation parameters between them (Szymon Rusinkiewicz, 2001)

\subsubsection{Data Processing:}

Point cloud processing means the process of transforming the raw registered point cloud into a final deliverable. These deliverables come in a wide variety of formats: cleaned point cloud data, standard 2D drawings, and fully 3D textured models for walkthrough animations. Often, scanner companies show impressive videos of point clouds turning into fully textured models in less than one second. However, in reality this process is still very time consuming and is mainly a manual process (S. Y. Lee, 2013). In the Figure 2.7 below, an overview is given of the different steps in the laser scanning process and their degree of automation.

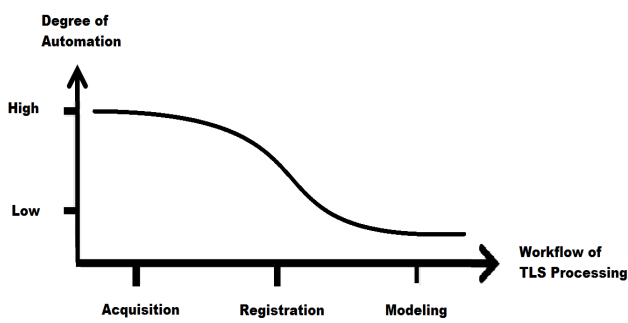

Figure 2.7: Shows degree of automation and TLS Workflow (A. Guren)

The result of a scan acquisition is a huge number of points in space, each having an $\mathrm{x}, \mathrm{y}, \mathrm{z}$ coordinate and usually a laser reflectance value. Some scanners even provide color information in the form of RGB values. The point cloud can be represented by drawing all these points on the screen, but this gives a very disordered impression and a user will have difficulties recognizing structures from the cloud. When every point is given its reflectance value or a color value, the overall structure becomes understandable (Ahmad Bazil, 2011).

After registration of these point clouds there are a lot of overlapping portions, which are redundant and make the future processes time consuming. Merging the point clouds operation will remove the overlapping portions. However, if the data is in the form of different registered point cloud than combining them will not remove the overlapping portions but it 
will reduce the memory usage. We have to use the "weld operation' to cut the overlapping parts and to join the point cloud.

In laser scanning, often high resolution photographic information is required for visualization of $3 \mathrm{D}$ models. For accurate mapping, the positions of the cameras and the internal camera settings (focal length, lens distortions...) have to be known in relation to the model. Commercial software packages provide algorithms that allow the user to manually select corresponding points between the image and the 3D model to determine these unknown parameters. Given enough correspondence, the position and parameters of the camera can be calculated. Mainly two different algorithms exist to project photographs onto the 3D model, texture mapping and texture draping. Texture draping can be described as putting the photograph onto a piece of elastic canvas and then pulling it over the 3D model (Naif Adel Haddad, 2011). This implies that areas that do not contain a lot of information or no information at all in the photograph (for instance very oblique surfaces) will be stretched and are therefore not textured correctly. Texture mapping overcomes this problem by first analyzing the visible parts in the image and then only projecting these photo pixels onto the $3 \mathrm{D}$ model. For the texture editing and mapping, visualization and rendering we have used evaluation version of Faro Scene software. The contribution of this work is design and implementation of Iterative Closest Point algorithm using three different techniques for optimizing atomization process. The novelty lies in the algorithm implementation process and this work provides first step towards 3D modeling of urban building for renovation, research, tourist, and education purpose.

\section{EXPERIMENTAL WORK \& RESULT}

Following Table 3.1 shows the comparative of ICP algorithm implemented with three BruteForce, kDTree, and Partial Overlap different technique with the results of CloudCompare open source tool.

\begin{tabular}{ccccc}
\hline Scan Set & BruteForce & kDTree & $\begin{array}{c}\text { Partial } \\
\text { Overlap }\end{array}$ & $\begin{array}{c}\text { Cloud } \\
\text { Compare }\end{array}$ \\
\hline $\begin{array}{c}\text { Scan_1 \& } \\
\text { Scan_2 }\end{array}$ & 1.5249 & $\mathbf{1 . 5 2 0 1}$ & 1.8126 & 2.51058 \\
\hline $\begin{array}{c}\text { Scan_3 \& } \\
\text { Scan_4 }\end{array}$ & 1.1313 & $\mathbf{1 . 1 1 9 5}$ & 1.2034 & 3.93242 \\
\hline $\begin{array}{c}\text { Scan_5 \& } \\
\text { Scan_6 }\end{array}$ & 2.2409 & 2.3069 & $\mathbf{2 . 2 3 0 4}$ & 2.43028 \\
Scan_7 \& & 0.6826 & 0.7301 & $\mathbf{0 . 6 8 0 6}$ & 0.79162 \\
Scan_8 & & & & \\
\hline $\begin{array}{c}\text { Scan_9 \& } \\
\text { Scan_10 }\end{array}$ & $\mathbf{0 . 6 0 7 4}$ & $\mathbf{0 . 6 4 4 8}$ & $\mathbf{0 . 6 5 0 3}$ & $\mathbf{0 . 6 9 7 7 5}$ \\
\hline Scan_11 \& & 0.7979 & $\mathbf{0 . 7 9 7 8}$ & 0.9238 & 1.27616 \\
Scan_12 & & & & \\
Max & $\mathbf{2 . 2 4 0 9}$ & $\mathbf{2 . 3 0 6 9}$ & $\mathbf{2 . 2 3 0 4}$ & $\mathbf{3 . 9 3 2 4 2}$ \\
Min & $\mathbf{0 . 6 0 7 4}$ & $\mathbf{0 . 6 4 4 8}$ & $\mathbf{0 . 6 5 0 3}$ & $\mathbf{0 . 6 9 7 7 5}$ \\
\hline Sum & $\mathbf{6 . 9 9}$ & $\mathbf{7 . 1 2}$ & $\mathbf{7 . 5 0}$ & $\mathbf{1 1 . 6 4}$ \\
\hline Average & $\mathbf{1 . 1 6 5}$ & $\mathbf{1 . 1 8 6 6}$ & $\mathbf{1 . 2 5}$ & $\mathbf{1 . 9 4}$ \\
\hline Accuracy & $\mathbf{9 8 . 8 3}$ & $\mathbf{9 8 . 8 1}$ & $\mathbf{9 8 . 7 5}$ & $\mathbf{9 8 . 0 6}$ \\
\hline
\end{tabular}

Table 3.1: Comparative results of algorithm with Open Source CloudCompare Tool.

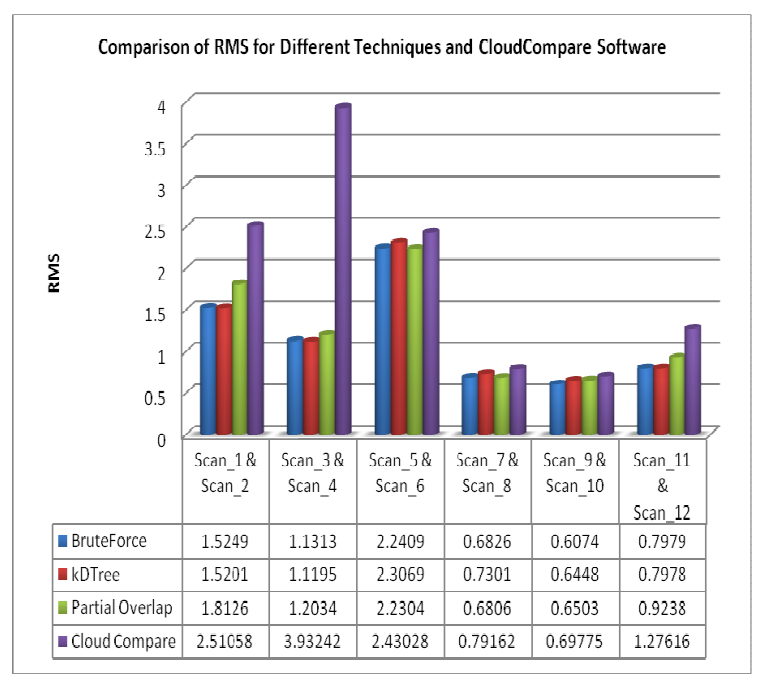

Figure 3.1: Comparison of RMS for Different Techniques and CloudCompare Software.

As we have shown in the Figure 3.1 results of Scan Registration also Table 3.1 shows comparative results with average accuracy 98.79 of implemented algorithm, and it is increased 0.73 as compared to open source CloudCompare software (98.06).

\section{ACKNOWLEDGEMENT}

Authors would like to thank to the Department of CS \& IT for their laboratory support, Department of Science \& Technology for financial assistant through fellowship provided under Srinivasa Ramanujan Geospatial Chair and the university authority for encouragement. And also LAB facility provided under UGC SAP (II) DRS Phase-I F.No.-3-42/2009;

\section{REFERENCES}

Ahmad Bazil bin Ashim, Zulkepli Majid, Halim Setan, 2011, "Three Dimensional Asset Documentation Using Terrestrial Laser Scanner Technology”, Geoinformation Science Journal, Vol. 11, No. 1, pp: 51-68.

Anne Chung Wei Lin., 2009, "Three Dimensional of Artefact Using Laser Scanner Vivid 910”, MSc. Thesis, University Teknologi Malaysia.

Beraldin, J.A., Blais, F., Boulanger, P., Cournoyer, L., Domey, J., El-Hakim, S.F., Godin, G., Rioux, M., Taylor, J., 2000, "Real World Modelling Through High Resolution Digital 3D Imaging of Objects and Structures", ISPRS Journal of Photogrammetry and Remote Sensing, 55, 230-250.

Besl, P. and Meckay, N. 1992, "A method for registration of 3-d shapes", IEEE Transaction on Pattern Analysis and Machine Intelligence, 14 No. 2:239-256.

Besl, Paul J, N.D. McKay, 1992, "A Method for Registration of 3-D Shapes", IEEE Trans. on Pattern Analysis and Machine Intelligence (Los Alamitos, CA, USA: IEEE Computer Society), 14 (2): 239-256. doi:10.1109/34.121791. 
Boehler, W., \& Marbs, A., 2004, "3D Scanning and Photogrammetry for Heritage Recording: A Comparison", Proceedings of 12th ICG.

Cabrelles, M., Galcera, S., Navarro, S., Lerma, J.L., Akasheh, T., \& Haddad, N., 2009, "Integration of 3D Laser Scanning, Photogrammetry and Thermography to Record Architectural Monuments", 22nd CIPA Symposium, Kyoto, Japan.

Chee Wei, Ong, Siew Chin, Cheong, Majid, Zulkepli, Setan, Halim, 2010, "3D documentation and preservation of historical monument using terrestrial laser scanning", Geoinformation Science Journal, 10 (1), pp. 73-90, ISSN 1511-9491.

Chen, Yang, Gerard Medioni, 1991, "Object modeling by registration of multiple range images", Image Vision Comput, (Newton, MA, USA: Butterworth-Heinemann): 145- 155, doi:10.1016/0262-8856(92)90066-C.

D. D. Lichti, M. P. Stewart, M. Tsakiri and A. J. Snow, 2000, "Calibration And Testing Of A Terrestrial Laser Scanner", International Archives of Photogrammetry and Remote Sensing. Vol. XXXIII, Part B5, Amsterdam.

El-Hakim S., Beraldin, A., Picard, M., 2002, "Detailed 3D Reconstruction of Monuments using Multiple Techniques", ISPRS/CIPA International Workshop on Scanning for Cultural Heritage Recording, Corfu, Greece, pp.58-64.

F. Remondino, S. El-Hakim, S. Girardi, A. Rizzi, S. Benedetti, L. Gonzo, 2011, "3D-ARCH2011 - 3D Virtual Reconstruction and Visualization of Complex Architectures", 4th International Workshop, Trento, Italy.

G. Vacca, M. Deidda, A. Dessi, M. Marras, 2012, "Laser Scanner Survey to Cultural Heritage Conservation and Restoration", International Archives of the Photogrammetry, Remote Sensing and Spatial Information Sciences, Volume XXXIX-B5, Australia.

Guarnier, A., Remondino, F., Vettore, A., 2006, "Digital photogrammetry and TLS data fusion applied to cultural heritage 3D modeling", IAP, RS \& SIS, www.photogrammetry.ethz.ch /general/ persons/fabio/Guarnieri _etal_ISPRSV 06.pdf.

Ismail Abd El hamid Mohamed, 2008, "Towards an Automatic Registration for Terrestrial Laser Scanner Data”, Ph.D. Thesis, University of Cairo, Egypt.

Library of Congress, accessed March 24, 2014, "Why Digital Preservation is Important for Everyone", Digital Archiving Resources, http://www.dar.cah.ucf.edu/items/show/64.

Lichti, D. D., Stewart, M. P., Tsakiri, M., Snow A. J., 2000, "Calibration and Testing of a Terrestrial Laser Scanner",
International Archives of Photogrammetry and Remote Sensing, Vol. XXXIII, Part B5, Amsterdam, Netherlands.

M. Hebel, U. Stilla, 2007, "Automatic Registration of Laser Point Clouds of Urban Areas", International Archives of Photogrammetry, Remote Sensing and Spatial Information Sciences, 36.

Naif Adel Haddad, 2011, "From ground surveying to 3D laser scanner: A review of techniques used for spatial documentation of historic sites", Journal of King Saud University Engineering Sciences.

Nik 'Azim Hazman Bin Nik Ab Hadi, Zulkepli Majid and Halim Setan, 2011, "Three Dimensional Modelling of Building using Faro Laser Scanner And AUTOCAD 2011 Software", Geoinformation Science Journal, Vol. 11, No. 2, pp: 1-16.

Nur Adlina Bt Ramli., 2010, "Three Dimensional Modeling of Historic Monument Using Topcon GLS-1000”, BEng.. Thesis. University Technology Malaysia.

Ong Chee Wei, Cheong Siew Chin, Zulkepli Majid, Halim Setan, 2010, "3D Documentation and Preservation of Historical Monument using Terrestrial Laser Scanning", Geoinformation Science Journal, Vol. 10, No. 1, pp: 73-90.

Ordóñez, C., Riveiro, B., Arias, P., Armesto, J., 2010, "Application of Close Range Photogrammetry to Deck Measurement in Recreational Ships.", http://www.mdpi.com /1424-8220/9/9/6991/pdf.

P. Dorninger, C. Nothegger, S. Rasztovits, 2013, "Efficient 3D Documentation of Neptune Fountain in the Park of Schönbrunn Palace at Millimeter Scale", ISPRS Annals of the Photogrammetry, Remote Sensing and Spatial Information Sciences, Volume II-5/W1, France.

Pfeifer, N., Dorninger, P., Haring, A. \& Fan, H., 2007, "Investigating Terrestrial Laser Scanning Intensity Data: Quality and Functional Relations", International Conference on Optical 3-D.

Remondino, F., \& El-Hakim, S., 2006, "Image based 3D Modeling: A Review", The Photogrammetric Record 21(115), 269-291.

Renyi Lium Guigang Shi, Fengquan Ji, 2010, “Ancient architecture reconstructing based on Terrestrial 3D Laser Scanning technology", Software Engineering and Data Mining (SEDM), China.

S. Y. Lee, Z. Majid, H. Setan, 2013, “3D Data Acquisition for Indoor Assets using Terrestrial Laser Scanning", ISPRS Annals of the Photogrammetry, Remote Sensing and Spatial Information Sciences, Turkey. 
Sajid Hussain, Håkan Grahn, 2007, "Fast kd-Tree Construction for 3D-Rendering Algorithms Like Ray Tracing", ISVC, Springer-Verlag Berlin Heidelberg, Part II, LNCS 4842, pp. 681-690.

Santala, J., Joana, V., 2010, "On the Calibration of a Groundbased Laser Scanner", www.fig.net/ /TS12_4_Santala_Joala. pdf.

Sequeira, V., Fiocco, M., Bostrom, G.. Gonçalves, J. G. M., 2003, “3D Verification of Plant Design", 25th ESARDA Symposium on Safeguards and Nuclear Material Management, Stockholm.

Singh, S., West, J., 1991, "Cyclone: A Laser Scanner for Mobile Robot Navigation", Carnegie Mellon University, Robotics Institute Technical Report, CMU-RI-TR-91-18.

Szymon Rusinkiewicz, Marc Levoy., 2001, "Efficient Variants of the ICP Algorithm", 3DIM, page 145-152, IEEE Computer Society.

W. Boehler, M. Bordas Vicent, A. Marbs., 2003, "Investigating Laser Scanner Accuracy", The XIXth CIPA Symposium at Antalya, Turkey.

Y. D. Rajendra, R. K. Dhumal, R. R. Manza, 2013, "Interior Renovation of an Urban Building using 3D Terrestrial Laser", International Journal of Advanced Research in Computer Science and Software Engineering (IJARCSSE), ISSN: 2277 128.

Yastikli, N., 2007, "Documentation of Cultural Heritage using Digital Photogrammetry and Laser Scanning", JCH, 8 (4), 423427.

Yonghuai Liu, 2006, "Automatic registration of overlapping 3D point clouds using closest points", Image and Vision Computing 24, Elsevier, 762-781.

Zhang, Zhengyou, 1994, "Iterative point matching for registration of free-form curves and surfaces", International Journal of Computer Vision (Springer), 13 (12): 119-152, doi:10. 1007/BF01427149. 